\title{
2s-Excitation and Photoionization of Neon Clusters
}

\author{
R. Flesch, ${ }^{1}$ N. Kosugi, ${ }^{2}$ A. Knop-Gericke, ${ }^{3}$ E. Rühl ${ }^{1}$ \\ ${ }^{1}$ Physikalische Chemie, Freie Universität Berlin, \\ Takustr 3, 14195 Berlin, Germany \\ ${ }^{2}$ Institute for Molecular Science, Myodaiji, Okazaki, 444-8585, Japan \\ ${ }^{3}$ Fritz-Haber-Institut der Max-Planck-Gesellschaft, Faradayweg 4-6, 14195 Berlin, Germany
}

\begin{abstract}
Variable size neon clusters consisting mostly of surface sites are investigated in the $\mathrm{Ne} 2 \mathrm{~s}$ regime (44-50 eV), which is characterized by Rydberg-/exciton-transitions. Distinct changes in peak shape are observed upon the transition from the atom to clusters as probed by yields of mass selected cations and threshold photoelectron spectra, where changes in coupling to the underlying $2 \mathrm{p}$ continuum are observed in clusters. Cluster size dependent changes cation yields are observed, which are rationalized in terms of a plausible autoionization mechanism, which involves $2 \mathrm{~s}$ ionization and is followed by charge delocalization via Interatomic Coulombic Decay (ICD). Size- and site-dependent changes in energy position of the Rydberg states as well as 2 s ionization energy are also observed. These results are interpreted along with model calculations which allow us to assign the experimental results in greater detail. The experimental and theoretical results are also used to deduce the exchange interaction of the Rydberg electron with the surrounding atoms and the induced polarization of the surrounding atoms in small neon clusters.
\end{abstract}




\section{Introduction}

Clusters have been investigated intensely during the last decades for determining structural and electronic properties as a function of size and composition [1]. Rare gas clusters are suitable model systems which are from the experimental point of view easy to produce and their size dependent structural and electronic properties are known from previous works [2-4]. Core level excitation has contributed significantly to this field, since element-selective excitation is provided as well as size- and site-specific properties were determined. This is due to characteristic energy shifts of the core exciton states relative to the atomic Rydberg states and shifts in core ionization energies [5-9]. The lowest energy Rydberg-/excitontransitions of clusters are significantly shifted to higher energy compared to the atomic transition, corresponding to a spectral blue-shift, whereas the higher ones are barely shifted or even show a spectral red-shift. Similar findings have also been reported for molecular clusters [10]. Earlier work has indicated that the exchange interaction of the Rydberg electron of the excited atom within a cluster with the surrounding atoms and the induced polarization of the surrounding atoms in the singly and doubly ionized atoms can be derived from the experimental results [11]. This provides the distinction between site-specific contributions of different surface locations in small clusters, corresponding to corner, edge, and face sites. These are also clearly distinguished from bulk sites [8,9]. Inner valence shell excitations of rare gas clusters, such as the Ar 3s-excitation, have also been investigated before [12-15]. They are characterized by a coupling to the strong valence continuum, as has been shown in previous work on argon clusters investigated near the $3 \mathrm{~s}$-excitation.

This provides the motivation for the present work, where we have studied the near-edge absorption in the $\mathrm{Ne} 2 \mathrm{~s}$-region via photoion yields as well as the $\mathrm{Ne} 2 \mathrm{~s}$ photoionization by threshold photoelectron spectroscopy (TPES). Pioneering work on the comparison of the absorption cross section of gaseous and solid neon indicates that distinct changes in electronic structure occur for 2s-excited neon [16]. The dominant Fano-profiles of the atom are shifted in energy and the corresponding exciton profiles of the solid are significantly broadened. The present work is also related to previous studies on $\mathrm{Ne} 2 \mathrm{~s}$ photoionization of dimers and clusters, which were discussed in terms of the Interatomic Coulombic Decay (ICD) in the $\mathrm{Ne}$ 2 s-regime [17-20]. Furthermore, $2 p$ correlation satellites in variable size neon clusters were investigated before by photoemission [21, 22]. Most recently, precise energy values of excitons and $2 \mathrm{~s}$ ionization energies were derived for the surface and for the bulk in condensed neon [23]. These results were compared to data on the isolated neon atom. 


\section{Experimental}

The experimental setup consists of a continuous jet expansion which is used for cluster preparation by adiabatic expansion. The jet is equipped with a $50 \mu \mathrm{m}$ nozzle separating the high pressure stagnation chamber from the vacuum in the expansion chamber. The typical stagnation pressure that is used in this work is 5 bar, where the stagnation temperature is varied between room temperature and $-185^{\circ} \mathrm{C}$. This leads to average cluster sizes $<\mathrm{N}>$, according to earlier size estimates [24], ranging between a purely atomic beam (room temperature) and up to $\sim 30$ [24] and $\sim 120$ [4] atoms per cluster, respectively. We use in the following the latter approach from ref. [4], which appears to give more realistic results considering the experimental results. The jet is skimmed, similar to previous work [5], and transferred into the interaction chamber, where it is crossed by a beam of monochromatic synchrotron radiation from the synchrotron radiation facility BESSY I (Berlin, Germany) at the U2-FSGM beamline [25]. The energy resolution of this beamline is adjusted by varying the slit width, ranging for the present work at $\mathrm{E} / \Delta \mathrm{E} \approx 3000$ (cf. [8]). Cations and electrons are detected alternatively by either a time-of-flight mass spectrometer (cf. [26]) or a threshold photoelectron spectrometer, which relies on angular discrimination of energetic electrons [27]. The experiments are performed in the multi-bunch mode of the storage ring. Cation yields are measured as a function of photon energy besides threshold photoelectron yields. Cation yields are obtained from pulsed cation extraction from the ionization region of the mass spectrometer by gating up to two cation channels simultaneously. This allows us to measure with high accuracy the energy shifts between resonant excitations into Rydberg states in atoms and exciton states in clusters, similarly to previous high-resolution work on atomic and molecular clusters [8-10]. Threshold photoelectron spectra are taken by using a continuous extraction field strength of $7.5 \mathrm{~V} / \mathrm{cm}$ in the ionization region. The analyzer has been used in earlier work [27]. The bandwidth of the photoelectrons is extracted from the atomic photoelectron spectra yielding $50 \pm 5 \mathrm{meV}$. Threshold photoelectron yields are obtained from selecting slow photoelectron while scanning the photon energy. Note, that the suppression of energetic electrons is somewhat less efficient than the detection of zero kinetic energy (ZEKE) photoelectrons, which requires single bunch operation of the storage ring along with pulsed extraction of the electrons from the ionization region [27].

\section{Model Calculations}

$A b$ initio quantum chemical calculations are carried out by using the GSCF3 code $[28,29]$ on $\mathrm{Ne}, \mathrm{Ne}_{15}$, and $\mathrm{Ne}_{19}$, similarly to the previous work on $\mathrm{N}_{2}$ and $\left(\mathrm{N}_{2}\right)_{13}$ [10]. The $\mathrm{Ne}_{15}$ (truncated 
$\mathrm{Ne}_{19}$ ) and $\mathrm{Ne}_{19}$ clusters are surface and bulk models based on the f.c.c. geometry having the first and second neighbor nearest atoms with a distance between the nearest Ne atoms of 3.06 $\AA$. The Ne 2s excited states are obtained from the improved virtual orbital or static exchange (STEX) method [30-33], which is applied to the fully relaxed SCF potentials for the localized $\mathrm{Ne} 2 \mathrm{~s}$ ionized states for $\mathrm{Ne}$ and central $\mathrm{Ne}$ in $\mathrm{Ne}_{15}$ and $\mathrm{Ne}_{19}$. The primitive basis functions, given by Huzinaga et al. [34], [73/7] are used as [721/52] for neutral neon atoms and [3111121/31111] for the ionized central Ne. The basis set is augmented for the ionized atom with five s $(\zeta=0.0253,0.01141,0.00589,0.00334$, and 0.00204), eight $\mathrm{p}(\zeta=0.044,0.0197$, 0.01013, 0.00573, 0.00349, 0.00224, 0.001052, and 0.0005), and six d $(\zeta=0.0282,0.01447$, $0.00817,0.00496,0.00319$, and 0.00214) diffuse functions in order to describe the $3 \mathrm{~s}-6 \mathrm{~s}, 3 \mathrm{p}$ - 6p, and 3d - 5d Rydberg states [35] and for the surrounding atoms with a $3 d$ polarization function $(\zeta=0.35)$. Electron correlation effects were not taken into account in the present calculations of $\mathrm{Ne}, \mathrm{Ne}_{15}$, and $\mathrm{Ne}_{19}$. Therefore, we apply a $0.829 \mathrm{eV}$ reduction of the calculated $\mathrm{Ne} 2 \mathrm{~s}$ threshold values $\mathrm{E}_{\text {th }}$ to $\mathrm{Ne}, \mathrm{Ne}_{15}$, and $\mathrm{Ne}_{19}$ and compare these results to the experimental findings.

\section{Results and Discussion}

Figure 1 shows a series of ion yield spectra of the dimer cation $\left(\mathrm{Ne}_{2}^{+}\right)$in comparison to the $\mathrm{Ne}^{+}$yield in the $\mathrm{Ne} 2 \mathrm{~s}$ regime $(45-49 \mathrm{eV})$. The atomic spectrum $\left(\mathrm{Ne}^{+}\right.$yield) is dominated by a series of resonances that are well-known from previous studies, in which the absorption and total or partial photoionization cross sections [36-39] were investigated. The resonant features that are superimposed to the $2 \mathrm{p}$ continuum can be described by the Rydberg formula [40]:

$$
E_{R S}=E_{t h}-R /(n-\delta)^{2}
$$

Here, $E_{R S}$ is the energy of the Rydberg state which can be described by the series formula, $E_{t h}$ is the threshold energy (or ionization energy), i.e. the convergence limit of the Rydberg series, $R$ is the Rydberg constant, $n$ is the principal quantum number, and $\delta$ is the quantum defect. The effective quantum number $n^{*}$ is given by $(\mathrm{n}-\delta)$. The term value $T$ is given by the energy difference $E_{t h}-E_{R S}$.

The dominating discrete features of the atom shown in Figure 1 are due to the $2 s^{1} 2 p^{6} n p$ Rydberg series, which are characterized by distinct Fano profiles, as pioneered before by Fano and coworkers [41, 42]. This Rydberg series is converging to the Ne 2s ionization 
energy $\left(2 s^{1} 2 p^{6}\right)$, which is found at 48.475 (cf. [21] and references cited therein). A weaker feature near $44.98 \mathrm{eV}$ is due to the atomic $2 \mathrm{p}^{4}\left({ }^{3} \mathrm{P}\right) 3 \mathrm{~s}\left({ }^{2} \mathrm{P}_{1 / 2,3 / 2}\right) 3 p$-transition [36]. In addition there are in the atomic spectrum weak features above $48.75 \mathrm{eV}$, which are assigned to $2 \mathrm{p}^{4}\left({ }^{3} \mathrm{P}\right) 3 \mathrm{~s}\left({ }^{2} \mathrm{P}_{1 / 2,3 / 2}\right) n p$-transitions $(\mathrm{n} \geq 6)$ and the atomic $2 \mathrm{p}^{4}\left({ }^{1} \mathrm{D}\right) 3 \mathrm{~s}\left({ }^{2} \mathrm{D}\right) 3 p$-transition [36].

The yield of the dimer cation undergoes distinct changes as the average cluster size $<\mathrm{N}>$ is increased. At the threshold of cluster formation $(<\mathrm{N}>=4)$ a weak $\mathrm{Ne}_{2}{ }^{+}$-signal is found, but this does not show any distinct features that are superimposed to the Ne $2 p$-continuum (see Figure 1). There might be some weak signal in the regime of the $2 s^{1} 2 p^{6} 4 p$-Rydberg transition near $47 \mathrm{eV}$. As a result, an entirely unstructured ion yield is recorded at $<\mathrm{N}>\leq 10$. This implies that for small clusters almost no distinct features are observed in the low intensity $\mathrm{Ne}_{2}{ }^{+}$-signal. This changes upon further increase in average cluster size, where at $<\mathrm{N}>=20$ three distinct resonances are observed (cf. Figure 1 and Table 1). These are assigned to Rydberg- or exciton-states of small clusters, of which the lowest one is assigned to the $2 s^{1} 2 p^{6} 4 p$-transition. The $2 s^{1} 2 p^{6} 3 p$-transition is not observed in the $\mathrm{Ne}_{2}{ }^{+}$yield for $<\mathrm{N}>=10$ and $<\mathrm{N}>=20$, even though it is by far the most intense transition in the spectrum of atomic neon (cf. bottom spectrum in Figure 1). The observed transitions $\left(2 s^{1} 2 p^{6} 4 p, 2 s^{1} 2 p^{6} 5 p\right.$, and $\left.2 s^{1} 2 p^{6} 6 p\right)$ are shifted in energy relative to the atomic transitions, where a red-shift is found that increases with increasing principal quantum number $n^{*}$ (cf. Table 1, where also the term values $T$ of the transitions are included). The energy values of these transitions are similar to those observed in previous work on condensed neon [23], underlining that these transitions are due to surface sites in clusters. The question arises, why such resonances do not occur at the threshold of cluster formation, i.e. at $<\mathrm{N}>\leq 10$ (cf. Figure 1). This question is related to the process that gives rise to the resonance that are also changed in excitation energy, shape, and width compared to the atomic features $[36,39]$. A suitable explanation comes from recent work [18], in which photoelectron spectra of neon clusters were discussed along with the Intramolecular Coulombic Decay (ICD). In the large cluster limit there is, unlike previous work on solid neon $[23,43]$, the 2 s-ionization energy red-shifted relative to the atomic value rather than blue-shifted. If the results on clusters [18] are correct, this would explain that the higher lying exciton states in clusters can be observed, which is not the case for the lowest one. This is due to the broadened peak shape of direct 2 s-ionization, which extends to ca. 1.5 $\mathrm{eV}$ below the atomic 2 s-ionization energy (cf. [18]), corresponding to $\approx 47 \mathrm{eV}$ photon energy). All resonances that are above this energy are enhanced in efficiency due to an autoionization process coupling to the 2 s-continuum that can only occur in sufficiently large clusters, which 
have a 2 s-ionization energy of the required red-shift. Therefore, the formation of fragment ions, which are detected in Figure 1, is not just reflecting the primary absorption process. It is rather assumed that autoionization enhances the primarily excited states in clusters, where the coupling to the 2 s-ionization continuum provides the observed size-dependence. Subsequently, charge de-localization occurs via ICD [18], an efficient channel that is already open for dimers above $43.3 \mathrm{eV}$ [17], so that singly charged cluster fragments are formed as observed in Figure 1. This explains the observation that no resonances are observed for $<\mathrm{N}>\leq$ 10 in the 2s-regime (cf. Figure 1). This cluster size-dependent process leading to cluster fragment ion formation explains also that the peak shape is different from the absorption features in atomic and condensed neon [16]. In solid neon it is known that Fano-profiles with a profile index $q$ of -1.6 occur, as was determined recently by Kassühlke and Feulner [23]. Clearly, the lowest exciton state is observed near $46.92 \mathrm{eV}$ in solid neon $[16,23]$ and there is no resemblance of such feature in clusters. By contrast, the cluster Rydberg-/excitonresonances show little coupling to the 2 s-continuum, pointing a different, size-dependent mechanism, which also involves substantial line broadening of $>100 \mathrm{meV}$. Several contributions may be considered in order to rationalize this finding, to which the excited-state lifetime may contribute most efficiently. Furthermore, the band width of the monochromator as well as different surfaces and bulk sites may contribute to the experimentally observed broadened line shapes, similar to previous work, where site-selective excitations in krypton clusters were discussed [8]. This aspect is discussed further below along with threshold photoelectron spectra.

The energy positions of the three distinct resonances are observed at $47.08 \mathrm{eV}, 47.54 \mathrm{eV}$, and $47.79 \mathrm{eV}$ (cf. Table 1). They are assigned to Ne $2 \mathrm{~s} \rightarrow n p$-transitions with $n=4,5$, and 6 . The width of these transitions is $130 \pm 10 \mathrm{meV}$, which is significantly broader than the atomic lines that are measured under the same conditions. This can be estimated e.g. from the asymmetric double excitation resonance $2 p^{4}\left({ }^{3} P\right) 3 \mathrm{~s} 3 p^{1} P_{o}$ at $44.980 \mathrm{eV}$ [39]. A line width of $25 \pm 5 \mathrm{meV}$ is observed for the present results, as shown for the $\mathrm{Ne}^{+}$yield in Figure 1. The energy resolution of the monochromator provides a broadening of $15 \mathrm{meV}$ (see Experimental Section) and the intrinsic broadening of this line is known to be of the order of $15 \mathrm{meV}$ [36]. Several reasons can be considered for such line broadening as briefly mentioned above, which may include different dynamics in clusters as compared to atoms, such as reduced excited-state lifetimes or blended features of several transitions that may occur in clusters. This aspect is analyzed in greater detail further below in the context of threshold photoelectron spectra. Clearly, the 
cluster Rydberg-/exciton-lines converge to the Ne $2 \mathrm{~s}$ ionization limit $\left(E_{t h}\right)$ in clusters that is extrapolated to be found at $48.24 \pm 0.01 \mathrm{eV}$, if the centers of the cluster exciton lines obtained from the $\mathrm{Ne}_{2}{ }^{+}$yield at constant quantum defect of 0.585 are used for $<\mathrm{N}>=90$. This indicates that the $\mathrm{Ne} 2$ s-ionization energy is substantially lowered compared to the atomic value (48.475 eV [21, 36], cf. Table 1), as also follows from the analysis of the threshold photoelectron spectra (see below). Considering that these values represent averaged values over all cluster sizes in the distribution and likely various surface sites, as was outlined in previous work $[8,9]$, a more detailed analysis of the present results is required. Furthermore, the extrapolated red-shift in $\mathrm{Ne} 2 \mathrm{~s}$ ionization energy in clusters at $<\mathrm{N}>=90$ is $0.24 \pm 0.01 \mathrm{eV}$ ( $\mathrm{E}_{\mathrm{th}}$ (cluster), see Table 1). A comparison with data from the literature indicates that for $<\mathrm{N}>=$ 100 a red-shift of $0.253 \mathrm{eV}$ was found for surface sites from XPS work [18]. The surface-tobulk red-shift is known to be for this cluster size $0.189 \mathrm{eV}$ [18], which would correspond to a $\mathrm{Ne} 2 \mathrm{~s}$ ionization energy for bulk sites of $48.033 \mathrm{eV}$. Evidently, the cluster exciton features shown in Figure 1 cannot be correlated to this convergence limit. This is another indication that the $\mathrm{Ne}_{2}^{+}$yield is essentially due to surface sites, despite the fact that there are at $<\mathrm{N}>=90$ also clusters containing bulk sites, as earlier XPS results indicate [18]. It should also be noted that for $<\mathrm{N}>>90$ the $\mathrm{Ne}_{2}{ }^{+}$yield gets weaker, since mostly small clusters contribute to this product channel, whereas larger ones stay intact and yield larger cationic fragments via single or double ionization. This is similar to earlier work on $\mathrm{Kr}$ clusters [8] and hints that the cluster size estimate according to ref. [4] is realistic. There are, however, distinct differences as compared to recent work on solid neon, which was studied via neon multilayers [23] (cf. Table 2). Similar to this work the surface and bulk Ne $2 \mathrm{~s} \rightarrow n p$ Rydberg series converging to the $2 \mathrm{~s}$ ionization energies were analyzed, yielding for the surface bound atoms $\mathrm{E}_{\text {th }}$ values of $47.9 \mathrm{eV}$ and $\mathrm{E}_{\mathrm{th}}=48.9 \mathrm{eV}$ for the bulk phase [23]. The values deduced for surface bound $\mathrm{Ne}$ are similar to those obtained from $\mathrm{Ne}_{2}^{+}$yields. A substantially different value is derived for the bulk phase, as compared to the results obtained from electron yields of large clusters [18]. This discrepancy will be further discussed in the following along with results from threshold photoelectron spectroscopy on neon clusters.

A series of threshold photoelectron spectra (TPES) is shown in Figure 2. We focus here on the spectral regime in which size-dependent changes occur, i.e. $46.7 \mathrm{eV}-49.2 \mathrm{eV}$. Note that there is no spectral feature due to clusters seen in the Ne $2 s \rightarrow 3 p$ regime. The bottom spectrum corresponds to the atomic spectrum, which shows low kinetic energy electrons that are primarily released at the $\mathrm{Ne} 2 \mathrm{~s}$ threshold, which is observed at $48.47 \pm 0.01 \mathrm{eV}$. This value 
is consistent with earlier work (cf. Table 1). Characteristic Fano-profiles are observed with low intensity below the $\mathrm{Ne} 2 \mathrm{~s}$ ionization threshold. These signals are due to a weak background of fast electrons. They come from autoionizing Rydberg states, such as $2 s^{1} 2 p^{6} 4 p\left({ }^{1} \mathrm{P}\right)$ and are detected by the threshold photoelectron spectrometer due to imperfect suppression of energetic electrons. Moreover, weak features, corresponding primarily to $2 s^{1} 2 p^{4}\left({ }^{3} \mathrm{P}\right) 3 s\left({ }^{2} \mathrm{P}_{1 / 2,3 / 2}\right) n p$-transitions [36], are observed above the Ne 2s-threshold between 48.5 and $49.0 \mathrm{eV}$.

As the cluster size is increased one observes distinct changes in the TPES, as shown in Figure 2. The most intense Rydberg-/exciton-features of clusters change their shape, broaden, and shift in energy, similar to the neon dimer cation yield $\mathrm{Ne}_{2}{ }^{+}$(cf. Figure 1). The energy positions of these features are consistent with the dimer cation yield (cf. Figure 1 and Table 1). A broad feature is observed between 48.0 and $48.5 \mathrm{eV}$. It gains intensity, as $<\mathrm{N}>$ is increased. It is assigned to the direct $\mathrm{Ne} 2 \mathrm{~s}$ ionization from clusters. It is significantly broader $(0.4 \pm 0.1 \mathrm{eV})$ than the atomic $2 \mathrm{~s}$-ionization $(0.05 \pm 0.01 \mathrm{eV})$. The ratio of the Rydberg-/excitonfeatures relative to the direct 2 s-ionization is enhanced as compared to the atom, indicating that electrons of low kinetic energy are released from clusters below the 2 s-threshold, as is expected due to inelastic scattering processes [27]. Both features are asymmetric in shape, but in a different way: (i) The atomic direct $2 \mathrm{~s}$ ionization shows the characteristic asymmetric shape that is due to the limited resolution of the threshold photoelectron analyzer (cf. ref. [44]). Symmetric line shapes have been obtained from low field strengths in the ionization region, which limits the sensitivity of the experimental approach, so that such conditions were not applied for the present work on clusters; (ii) the direct $\mathrm{Ne} 2 \mathrm{~s}$ ionization signal of clusters is steeper at the high energy side and it merges into the Rydberg-/exciton-regime, as is most clearly visible from the spectrum taken at $<\mathrm{N}>=140$. This is rationalized in terms of different dynamics as well as different sites contributing to the direct ionization in TPES spectra, similar to earlier work on photoionization of homogeneous and heterogeneous rare gas clusters $[9,11,45]$. There, it was noticed that not just surface- and bulk-sites can be distinguished, rather than that different surface-sites contribute to slightly different ionization energies. This is mostly due to different polarization shifts that are related to the number of nearest neighbors to the absorbing and ionized site. The highest energy contribution that is least shifted relative to the isolated atom is due to corner-sites, if dimers are neglected (cf. [9]). This is due to the fact that corner sites have the lowest number of nearest neighbors. They are known to be weak in intensity, since they mostly occur in small clusters, whereas in 
larger clusters corner-sites are the weakest surface component [8]. The most intense contribution to the surface signal has been found for edge-sites [9, 11]. They occur slightly red-shifted in energy relative to corner-sites for all cluster sizes, especially for those clusters of imperfect shapes, i.e. clusters containing surface defects by which face-sites are converted to edge-sites. Finally, face-sites can only occur, if the clusters are sufficiently large in size. Contributions of face-sites are more shifted to lower binding energy than the other surfacesites. This implies for the size evolution shown in Figure 2 that the $\mathrm{Ne} 2 \mathrm{~s}$ ionization signal changes its shape and energy position as the cluster size is increased. At $<\mathrm{N}>=6$ the mixing ratio of clusters is weak, so that this region appears to be almost unchanged compared to the atom. At $\langle\mathrm{N}\rangle=30$ there is a distinct maximum at $48.35 \mathrm{eV}$ that is red-shifted by $120 \pm 10$ $\mathrm{meV}$ relative to the atomic value. It is weak in intensity and tails out down to $48 \mathrm{eV}$ as a result of weak contributions of larger clusters. At $<\mathrm{N}>=140$ this maximum is further red-shifted by $60 \pm 10 \mathrm{meV}$ and the intensity down to $47.9 \mathrm{eV}$ has significantly increased. An even more clear picture is obtained from subtracting the atomic contribution from the TPES taken at $<\mathrm{N}\rangle=$ 140, which corresponds to the top spectrum shown in Figure 2. Note that the region of the atomic signal was not considered, since this dominating signal in all TPES leads to artefacts upon subtraction, so that these points were erased and their intensity was interpolated to the neighboring points that were not affected. The energy positions of the cluster Rydberg/exciton-bands that are derived in this way are similar to those shown in Figure 1, but their energy positions are slightly different (see Table 1). The top spectrum of Figure 2 is used for further analysis of the cluster Rydberg-/exciton-bands. The result of this analysis is shown in Figure 3. Each of the cluster features is de-convoluted by three Gaussians of $66 \mathrm{meV}$ width, yielding a resemblance with the experimental results. Other line shapes, such as Voigt profiles, were tried, but did not improve the quality of the fit of the experimental results. The results are listed in Table 1. For all three Rydberg-/exciton-features, i.e. Ne $2 \mathrm{~s} \rightarrow 4 p, 5 p, 6 p$, the central contribution is strongest (cf. Figure 3). It is assigned to edge-sites in neon clusters. The weaker ones are observed either at lower or higher energy. These are due to face- and corner-sites, respectively in accordance to earlier work on rare gas clusters [9]. Deviations in intensity for the surface-sites are observed for these transitions. Clearly, the Ne $2 \mathrm{~s} \rightarrow 4 p$ transition shows higher intensity for the corner- and face-sites than the $\mathrm{Ne} 2 \mathrm{~s} \rightarrow 5 p$ - and $\mathrm{Ne}$ $2 s \rightarrow 6 p$-transitions. The origin of these variations in intensity might be due to different broadening mechanisms of state-dependent dynamics, which evidently affects the lowest energy transition more than the other ones. 
The discrete features below the 2s-ionization energy show indeed that they can be fitted by the Rydberg formula (eq. (1)) with almost constant quantum defect providing the convergence limits $E_{t h}$ that are indicated by vertical lines in Figure 3. These range between $48.17 \pm 0.01 \mathrm{eV}$ (face-sites), $48.23 \pm 0.01 \mathrm{eV}$ (edge-sites), and $48.28 \pm 0.01 \mathrm{eV}$ (corner-sites) (cf. Table 1). The ionization energy of the main component (edge-sites, $48.23 \pm 0.01 \mathrm{eV}$ ) is in agreement with that obtained from the $\mathrm{Ne}_{2}{ }^{+}$-yield $(48.24 \pm 0.01 \mathrm{eV})$, where the data analysis error $( \pm 0.01 \mathrm{eV})$ is taken into account. The results shown in Figure 3 clearly indicate that the most intense portion of the Ne 2 s ionization signal is due to different surface-sites.

Note that we did not attempt to fit the broad signal corresponding to direct $\mathrm{Ne} 2 \mathrm{~s}$ ionization. This was due to the following reasons: (i) earlier work on clusters has indicated that the line shapes in clusters are quite different from those of the isolated atom [27] increasing the number of unknown parameters; (ii) in the regime between the discrete resonances and the maximum of the direct ionization signal there is also TPES-intensity, which may have contributions from bulk-sites as well as higher-lying Rydberg-/exciton-states. These cannot be easily distinguished from the experimental results. Evidence for bulk-sites is not clearly observed for the distinct Rydberg-/exciton-bands as well as for the direct $\mathrm{Ne} 2 \mathrm{~s}$ ionization, where one would expect at $0.5-0.6 \mathrm{eV}$ below the atomic $\mathrm{Ne} 2 \mathrm{~s}$ ionization energy a signal in TPES [18], which is evidently not observed in Figures 2 and 3 with high intensity. This underlines that primarily clusters containing surface-sites are present in the jet. Furthermore, we also do not find evidence for a $\mathrm{Ne} 2 \mathrm{~s}$ ionization signal at higher energy, as was deduced from recent work on Ne multilayers [23]. There, we only observe that the discrete double excitation features between $48.7 \mathrm{eV}$ and $49.0 \mathrm{eV}$ (cf. ref. [36]) vanish in clusters.

Further support for the assignments that are based on experimental results comes from calculations. Table 2 shows results from calculations of the Ne 2 s ionization energies as well as the $\mathrm{Ne} 2 \mathrm{~s} \rightarrow 3 \mathrm{p}, 4 \mathrm{p}, 5 \mathrm{p}$, and 6p Rydberg-/exciton-transitions of free neon atoms and clusters. Effective quantum numbers $\mathrm{n}^{*}$ are included in order to indicate that the observed transitions can be described by the Rydberg formula (cf. eq. (1)). We have used as a model plausible structures of clusters with f.c.c. geometry (first and second shell). The central atoms of $\mathrm{Ne}_{15}$ (truncated $\mathrm{Ne}_{19}$ ) and $\mathrm{Ne}_{19}$ correspond to surface and bulk properties of neon. Note that the results contained in Table 2 refer to the surface and bulk of clusters, whereas the experimental results from clusters are de-convoluted into various surface components, i.e. face-, edge-, and corner-sites. In the calculations on neon clusters, the $2 \mathrm{~s}$ ionization energy is 
by $0.23 \mathrm{eV}$ (surface) and $0.32 \mathrm{eV}$ (bulk) red-shifted relative to the free atom, whereas the $\mathrm{Ne}$ $2 \mathrm{~s} \rightarrow 3 \mathrm{p}$ excitation energy is blue-shifted by $0.72 \mathrm{eV}$ (surface) and $0.81 \mathrm{eV}$ (bulk) relative to the atomic value. Such shifts have been discussed before for determining the exchange interaction of the Rydberg electron with the nearest neighbor sites in clusters [11]. This provides a site- and state-dependence of the exchange interaction. The Rydberg electron may be confined within the cage which is composed of the nearest neighbors. The significant blueshift of the lowest Rydberg states in clusters gives an indication that the exchange interaction energy $E_{E X}$ between the Rydberg electron and the nearest neighbor atoms in the surrounding sites is larger than the polarization, which is induced by the singly ionized atom after core level excitation. Previous work has shown that one can determine the exchange interaction in core-excited singly charged species, according to the simple expression [11]:

$$
E_{E X}=\Delta E_{X A S}-E_{i P L}
$$

Here, $\triangle E_{X A S}$ is the energy shift of the transition in clusters relative to the atomic value and $E_{i P L}$ is the induced polarization energy, which is obtained from the shift of the ionization energy $\mathrm{E}_{\text {th }}$ of the cluster relative to the atomic value. The exchange energy $E_{E X}$ is included both in Table 1, where it refers to experimental values, and in Table 2, where the calculated values are considered. Specifically, this means that the exchange interaction of the $3 p$ Rydberg electron is estimated by model simulations to be $0.95 \mathrm{eV}$ (surface) and $1.13 \mathrm{eV}$ (bulk). It is derived from the sum of the calculated energy differences of (i) the blue-shift of the Ne $2 \mathrm{~s} \rightarrow$ $3 p$ transition in clusters compared to the atomic transition, yielding $0.72 \mathrm{eV}$ (surface) and 0.81 $\mathrm{eV}$ (bulk), respectively, and (ii) the difference in Ne 2s ionization energy of clusters and the atom $E_{\text {th }}$ (cluster) $-E_{t h}($ atom) $=-0.23 \mathrm{eV}$ (surface) and $-0.32 \mathrm{eV}$ (bulk). This value is consistent with that derived from the experimental results, which yields $0.97 \mathrm{eV}$ (cf. Table 1) for the signal that comes from the $\mathrm{Ne}^{+}$and $\mathrm{Ne}_{2}^{+}$yield, which is the sum of $0.73 \mathrm{eV}$ and $0.24 \mathrm{eV}$ (cf. Table 1). On the other hand, the calculated Ne $2 s \rightarrow 4 p$ excitation energy is slightly blueshifted by $0.12 \mathrm{eV}$ (surface) and $0.17 \mathrm{eV}$ (bulk) leading to a lower exchange interaction of $0.35 \mathrm{eV}$ (surface) and $0.49 \mathrm{eV}$ (bulk), so that the exchange energy is lowered (see Table 2). This is expected since the $4 p$-Rydberg orbital is larger than the $3 p$-orbital, implying a decreased interaction with electrons of neighboring atoms in clusters. Consistently, the experimental value of the exchange energy of the Ne $2 \mathrm{~s} \rightarrow 4 \mathrm{p}$ transition is significantly lowered from $0.97 \mathrm{eV}$ (3p) to $0.20 \mathrm{eV}$ (cf. Table 1), which is similarly also observed in $\mathrm{Kr}$ and Xe clusters [11]. Even lower values are observed for the higher Rydberg-/exciton- 
transitions, indicating that the interaction between the Rydberg electron and neighboring atoms in cluster becomes smaller the larger the Rydberg orbital is. Clearly, for the $\mathrm{Ne} 2 \mathrm{~s} \rightarrow$ 6 -transition the exchange energy becomes negligibly small, as follows consistently from the experimental and modeled results shown in Tables 1 and 2.

\section{Conclusions}

The Ne 2s regime has been investigated regarding size effects in electronic structure of weakly bound clusters. It is different from other absorption edges that have been studied before, providing element-selective excitation, since there is a strong underlying $2 \mathrm{p}$ continuum including interactions with the atomic Rydberg states leading to Fano profiles. It is shown that in clusters the Rydberg-/exciton-resonances show a smaller coupling to the $2 \mathrm{p}$ continuum than the atom, which is also different from the absorption of condensed neon. These changes are discussed in terms of a cluster-size dependent ionization and fragmentation mechanism, which involves autoionization along with 2 s ionization and subsequent charge separation via Interatomic Coulombic Decay (ICD) leading to the observed species, i.e. yields of cluster cations and threshold photoelectrons. The resonances are also used to determine the $2 \mathrm{~s}$ ionization energies $\left(E_{t h}\right)$ in clusters. These results are compared to model calculations as well as experimental threshold photoelectron spectra providing consistent information on the exchange interaction energy $E_{E X}$ between the Rydberg electron with the neighboring sites in clusters. Contributions for various surface sites, i.e. corners, edges, and faces, are identified and their $2 \mathrm{~s}$ ionization energies are identified. The present results are compared to previous work on variable size clusters as well as multilayers of condensed neon, providing novel insights into size effects of variable size clusters.

\section{Acknowledgments}

Financial support by the German Research Foundation (DFG, contract no.: RU 420/8-1) is gratefully acknowledged.

\section{References}

[1] H. Haberland, Clusters of Atoms and Molecules (Springer, Berlin, 1994).

[2] J. Farges, M. F. Deferaudy, B. Raoult, and G. Torchet, Surf. Sci. 106 (1981) 95.

[3] J. Farges, M. F. Deferaudy, B. Raoult, and G. Torchet, J. Chem. Phys. 78 (1983) 5067.

[4] J. Farges, M. F. Deferaudy, B. Raoult, and G. Torchet, J. Chem. Phys. 84 (1986) 3491.

[5] E. Rühl, C. Heinzel, A. P. Hitchcock, and H. Baumgärtel, J. Chem. Phys. 98 (1993) 2653.

[6] O. Björneholm, F. Federmann, F. Fössing, and T. Möller, Phys. Rev. Lett. 74 (1995) 3017. 
[7] E. Rühl, Int. J. Mass Spectrom. 229 (2003) 117.

[8] A. Knop, B. Wassermann, and E. Rühl, Phys. Rev. Lett. 80 (1998) 2302.

[9] T. Hatsui, H. Setoyama, N. Kosugi, B. Wassermann, I. L. Bradeanu, and E. Rühl, J. Chem. Phys. 123 (2005) 154304.

[10] R. Flesch, N. Kosugi, I. L. Bradeanu, J. J. Neville, and E. Rühl, J. Chem. Phys. 121 (2004) 8343.

[11] M. Nagasaka, T. Hatsui, H. Setoyama, E. Rühl, and N. Kosugi, J. Electron Spectrosc. Relat. Phenom. 183 (2011) 29.

[12] M. White and J. Grover, J. Chem. Phys. 79 (1983) 4124.

[13] R. Müller, M. Joppien, and T. Möller, Z. Phys. D - Atoms, Molecules and Clusters 26 (1993) 370.

[14] A. A. Pavlychev and E. Rühl, J. Electron Spectrosc. Relat. Phenom. 106 (2000) 207.

[15] H. Zhang, D. Rolles, J. D. Bozek, and N. Berrah, J. Phys B: At. Mol. Opt Phys. 42 (2009) 105103.

[16] R. Haensel, N. Kosuch, U. Nielsen, U. Rössler, and B. Sonntag, Phys. Rev. B 7 (1973) 1577.

[17] T. Jahnke, A. Czasch, M. S. Schöffler, S. Schössler, A. Knapp, M. Käsz, J. Titze, C. Wimmer, K. Kreidi, R. E. Grisenti, A. Staudte, O. Jagutzki, U. Hergenhahn, H. Schmidt-Böcking, and R. Dörner, Phys. Rev. Lett. 93 (2004) 163401.

[18] G. Öhrwall, M. Tchaplyguine, M. Lundwall, R. Feifel, H. Bergersen, T. Rander, A. Lindblad, J. Schulz, S. Peredkov, S. Barth, S. Marburger, U. Hergenhahn, S. Svensson, and O. Björneholm, Phys. Rev. Lett. 93 (2004) 173401.

[19] S. Bahrdt, S. Marburger, O. Kugeler, V. Ulrich, S. Joshi, A. M. Bradshaw, and U. Hergenhahn, Chem. Phys. 329 (2006) 246.

[20] S. Scheidt, V. Averbukh, H.-D. Meyer, N. Moiseyev, R. Santra, T. Sommerfeld, J. Zobeley, and L. S. Cederbaum, J. Chem. Phys. 121 (2004) 8393.

[21] S. Joshi, S. Bahrdt, S. Marburger, V. Ulrich, and U. Hergenhahn, Phys. Rev. B 73 (2006) 235404.

[22] U. Hergenhahn, A. Kolmakov, M. Riedler, A. R. B. de Castro, O. Löfken, and T. Möller, Chem. Phys. Lett. 351 (2002) 235.

[23] B. Kassühlke and P. Feulner, Low Temp. Phys. 38 (2012) 749.

[24] R. Karnbach, M. Joppien, J. Stapelfeldt, J. Wörmer, and T. Möller, Rev. Sci. Instrum. 64 (1993) 2838.

[25] J. Bahrdt, A. Gaupp, W. Gudat, M. Mast, K. Molter, W. B. Peatman, M. Scheer, T. Schroeter, and C. Wang, Rev. Sci. Instrum. 63 (1992) 339.

[26] E. Rühl, C. Heinzel, H. Baumgärtel, and A. P. Hitchcock, Chem. Phys. 169 (1993) 243.

[27] A. Knop, H. W. Jochims, A. L. D. Kilcoyne, A. P. Hitchcock, and E. Rühl, Chem. Phys. Lett. 223 (1994) 553.

[28] N. Kosugi and H. Kuroda, Chem. Phys. Lett. 74 (1980) 490.

[29] N. Kosugi, Theor. Chim. Acta 72 (1987) 149.

[30] W. J. Hunt and W. A. Goddard III, Chem. Phys. Lett. 3 (1969) 414.

[31] S. Iwata, N. Kosugi, and O. Nomura, Jpn. J. Appl. Phys. 17-S2, (1978) 109.

[32] N. Kosugi, E. Shigemasa, and A. Yagishita, Chem. Phys. Lett. 190 (1992) 481.

[33] H. Ågren, V. Carravetta, O. Vahtras, and L. G. M. Pettersson, Chem. Phys. Lett. 222 (1994) 75 .

[34] S. Huzinaga, J. Andzelm, M. Klobukowski, E. Eadzio-Andzelm, Y. Sakai, and H. Tatewaki, Gaussian Basis Sets for Molecular Calculations (Elsevier, Amsterdam, 1984).

[35] K. Kaufmann, C. Nager, and M. Jungen, Chem. Phys. 95 (1985) 385. 
[36] K. Schulz, M. Domke, R. Püttner, A. Gutierrez, G. Kaindl, G. Miecznik, and C. H. Greene, Phys. Rev. A 54 (1996) 3095.

[37] S. E. Canton, A. A. Wills, T. W. Gorczyca, E. Sokell, J. D. Bozek, G. Turri, M. Wiedenhoeft, X. Feng, and N. Berrah, J. Phys. B: At. Mol. Opt. Phys. 36 (2003) L181.

[38] B. Langer, N. Berrah, R. Wehlitz, T. W. Gorczyca, J. Bozek, and A. Farhat, J. Phys. B: At. Mol. Phys. 30 (1997) 593.

[39] K. Codling, R. P. Madden, and D. L. Ederer, Phys. Rev. 155 (1967) 26.

[40] J. Berkowitz, Photoabsorption, Photoionization and Photoelectron Spectroscopy (Academic Press, New York, 1979).

[41] U. Fano, Phys. Rev. 124 (1961) 1866.

[42] U. Fano and J. W. Cooper, Phys. Rev. 137 (1965) 1364.

[43] P. Wiethoff, H. U. Ehrke, D. Menzel, and P. Feulner, Phys. Rev. Lett. 74 (1995) 3792.

[44] W. B. Peatman, G. B. Kasting, and D. J. Wilson, J. Electron Spectrosc. Relat. Phenom. 7 (1975) 233.

[45] M. Nagasaka, N. Kosugi, and E. Rühl, J. Chem. Phys. 136 (2012) 234312. 
Table 1: Experimental Ne $2 s \rightarrow n p$ transition energies $\left(E_{X A S}\right)$, energy shifts between atom and cluster $\left(\Delta E_{X A S}\right)$, exchange interaction energies $\left(E_{E X}\right)$, term values $(T)$, and effective quantum numbers $\left(n^{*}\right)$ for atomic neon and neon clusters at $<\mathrm{N}>=140$ (error limit for energy values: $\pm 0.01 \mathrm{eV}$ ). Site-resolved results given for clusters are derived from a de-convolution of the experimental TPES signals into face, edge, and corner contributions (see Figure 3 and text for further details). The "experimental" transitions refer to the $\mathrm{Ne}^{+}$-yield (atom) or the $\mathrm{Ne}_{2}{ }^{+}$-yield (clusters) (see Figure 1).

\begin{tabular}{|c|c|c|c|c|c|c|}
\hline $\begin{array}{l}\text { Transitio } \\
\text { n } \\
\end{array}$ & Species & $\begin{array}{l}E_{X A S} \\
{[\mathrm{eV}]} \\
\end{array}$ & $\begin{array}{c}\Delta E_{X A S} \\
{[\mathrm{eV}]}\end{array}$ & $\begin{array}{r}E_{E X} \\
{[\mathrm{eV}]} \\
\end{array}$ & $\begin{array}{c}T \\
{[\mathrm{eV}]}\end{array}$ & $n^{*}$ \\
\hline \multirow[t]{2}{*}{$2 s \rightarrow 3 p$} & atom & $45.54^{\mathrm{a}}$ & & & 2.94 & 2.15 \\
\hline & cluster & $46.27^{\mathrm{a}}$ & 0.73 & 0.97 & 1.97 & 2.63 \\
\hline \multirow[t]{5}{*}{$2 s \rightarrow 4 p$} & $\begin{array}{l}\text { atom } \\
\text { cluster }\end{array}$ & $47.12^{\mathrm{a}}$ & & & 1.36 & 3.17 \\
\hline & experimental & $47.08^{\mathrm{a}}$ & -0.04 & 0.20 & 1.16 & 3.42 \\
\hline & face & 46.99 & -0.13 & 0.18 & 1.18 & 3.40 \\
\hline & edge & 47.08 & -0.04 & 0.21 & 1.15 & 3.44 \\
\hline & corner & 47.14 & 0.02 & 0.22 & 1.14 & 3.45 \\
\hline \multirow[t]{5}{*}{$2 s \rightarrow 5 p$} & $\begin{array}{l}\text { atom } \\
\text { cluster }\end{array}$ & $47.70^{\mathrm{a}}$ & & & 0.78 & 4.19 \\
\hline & experimental & $47.54^{\mathrm{a}}$ & -0.16 & 0.08 & 0.70 & 4.41 \\
\hline & face & 47.44 & -0.26 & 0.05 & 0.73 & 4.32 \\
\hline & edge & 47.54 & -0.16 & 0.09 & 0.69 & 4.44 \\
\hline & corner & 47.59 & -0.11 & 0.09 & 0.69 & 4.44 \\
\hline \multirow[t]{5}{*}{$2 s \rightarrow 6 p$} & $\begin{array}{l}\text { atom } \\
\text { cluster }\end{array}$ & $47.99^{\mathrm{a}}$ & & & 0.49 & 5.30 \\
\hline & experimental & $47.79^{\mathrm{a}}$ & -0.20 & 0.04 & 0.45 & 5.50 \\
\hline & face & 47.70 & -0.29 & 0.02 & 0.47 & 5.38 \\
\hline & edge & 47.77 & -0.22 & 0.03 & 0.46 & 5.44 \\
\hline & corner & 47.82 & -0.17 & 0.03 & 0.46 & 5.44 \\
\hline \multirow[t]{5}{*}{$\overline{E_{t h}}$} & $\begin{array}{l}\text { atom } \\
\text { cluster }\end{array}$ & $48.475^{b}$ & & & & \\
\hline & experimental & $48.24^{\mathrm{a}}$ & $-0.24^{c}$ & & & \\
\hline & face & 48.17 & $-0.31^{c}$ & & & \\
\hline & edge & 48.23 & $-0.25^{c}$ & & & \\
\hline & corner & 48.28 & $-0.20^{c}$ & & & \\
\hline
\end{tabular}


Table 2: Calculated energies for $\mathrm{Ne} 2 \mathrm{~s} \rightarrow n p$-transitions ( $E_{X A S}($ calc.)), energy shifts between atom and cluster $\left(\triangle E_{X A S}\right)$, exchange energy $\left(E_{E x}\right)$, term values $(T)$, effective quantum numbers $\left(n^{*}\right)$, reference values (Ref.) and energy shifts between the atom and clusters in reference values $\left(\Delta E_{R e f}\right)$. Calculated term values and effective quantum numbers refer to $\mathrm{Ne}_{19}$ and truncated $\mathrm{Ne}_{19}\left(\mathrm{Ne}_{15}\right) . E_{t h}$ : $\mathrm{Ne} 2 \mathrm{~s}$ ionization energy.

\begin{tabular}{|c|c|c|c|c|c|c|c|c|}
\hline Transition & Species & $\begin{array}{c}E_{X A S}(\text { calc. })^{\mathrm{e}} \\
{[\mathrm{eV}]}\end{array}$ & $\begin{array}{c}\Delta E_{X A S} \\
{[\mathrm{eV}]}\end{array}$ & $\begin{array}{l}E_{E x} \\
{[\mathrm{eV}]}\end{array}$ & $\begin{array}{c}T \\
{[\mathrm{eV}]}\end{array}$ & $n^{*}$ & $\begin{array}{l}\text { Ref. } \\
{[\mathrm{eV}]}\end{array}$ & $\begin{array}{c}\Delta E_{R e f} \\
{[\mathrm{eV}]}\end{array}$ \\
\hline \multirow[t]{3}{*}{$2 s \rightarrow 3 p$} & $\begin{array}{l}\text { atom } \\
\text { cluster }\end{array}$ & 45.83 & & & 2.65 & 2.27 & $45.544^{\mathrm{d}}$ & \\
\hline & surface & 46.55 & 0.72 & 0.95 & 1.70 & 2.83 & $46.32^{b}$ & $0.78^{b}$ \\
\hline & bulk & 46.64 & 0.81 & 1.13 & 1.52 & 2.99 & $46.85^{b}$ & $1.31^{\mathrm{b}}$ \\
\hline \multirow[t]{3}{*}{$2 s \rightarrow 4 p$} & $\begin{array}{l}\text { atom } \\
\text { cluster }\end{array}$ & 47.15 & & & 1.33 & 3.20 & $47.1193^{d}$ & \\
\hline & surface & 47.27 & 0.12 & 0.35 & 0.98 & 3.73 & $47.11^{\mathrm{b}}$ & $-0.01^{b}$ \\
\hline & bulk & 47.32 & 0.17 & 0.49 & 0.84 & 4.03 & $48.00^{\mathrm{b}}$ & $0.88^{\mathrm{b}}$ \\
\hline \multirow[t]{3}{*}{$2 s \rightarrow 5 p$} & $\begin{array}{l}\text { atom } \\
\text { cluster }\end{array}$ & 47.71 & & & 0.77 & 4.22 & $47.6952^{d}$ & \\
\hline & surface & 47.63 & -0.08 & 0.15 & 0.62 & 4.68 & $47.45^{\mathrm{b}}$ & $-0.25^{b}$ \\
\hline & bulk & 47.61 & -0.10 & 0.22 & 0.55 & 4.99 & $48.44^{b}$ & $0.74^{b}$ \\
\hline \multirow[t]{3}{*}{$2 s \rightarrow 6 p$} & $\begin{array}{l}\text { atom } \\
\text { cluster }\end{array}$ & 47.98 & & & 0.50 & 5.24 & $47.9650^{\mathrm{d}}$ & \\
\hline & surface & 47.80 & -0.18 & 0.05 & 0.45 & 5.50 & $47.65^{b}$ & $-0.32^{b}$ \\
\hline & bulk & 47.79 & -0.19 & 0.13 & 0.37 & 6.09 & $48.70^{b}$ & $0.74^{\mathrm{b}}$ \\
\hline \multirow[t]{5}{*}{$E_{t h}$} & $\begin{array}{l}\text { atom } \\
\text { cluster }\end{array}$ & $48.475^{\mathrm{a}}$ & & & & & & \\
\hline & surface & 48.250 & $-0.23^{f}$ & & & & $48.22^{\mathrm{c}}$ & $-0.26^{\mathrm{c}}$ \\
\hline & $\begin{array}{l}\text { bulk } \\
\text { condensed } \\
\text { neon }\end{array}$ & 48.157 & $-0.32^{f}$ & & & & $48.03^{c}$ & $-0.45^{\mathrm{c}}$ \\
\hline & surface & & & & & & $47.90^{\mathrm{b}}$ & $-0.58^{b}$ \\
\hline & bulk & & & & & & $48.90^{\mathrm{b}}$ & $0.43^{b}$ \\
\hline
\end{tabular}

a taken from refs. [21, 36] and references cited therein.

b taken from solid neon according to ref. [23].

c taken from ref. [18] for $<\mathrm{N}>=100$.

d taken from ref. [36].

e All calculated ionization $\left(E_{t h}\right)$ and excitation energies $\left(E_{X A S}(\right.$ calc. $\left.)\right)$ are reduced by $0.829 \mathrm{eV}$ (see text for further details).

${ }^{\mathrm{f}}$ This corresponds to the stabilization energy by the induced polarization effect, $E_{i P L}$. 


\section{Figure Captions}

\section{Figure 1:}

Photoion yield curves of the neon dimer $\left(\mathrm{Ne}_{2}{ }^{+}\right)$as a function of photon energy near the Ne 2sabsorption as a function of the average cluster size $<\mathrm{N}>$. The $\mathrm{Ne}^{+}$yield, corresponding to the atomic spectrum, is shown for a comparison (bottom spectrum).

\section{Figure 2:}

Threshold photoelectron spectra (TPES) of neon clusters as a function of the average cluster size $<\mathrm{N}>$. A threshold photoelectron spectrum of the atom is shown for a comparison. The top spectrum shows the TPES of neon clusters at $<\mathrm{N}>=140$, where the atomic contribution has been subtracted.

\section{Figure 3:}

Detailed analysis of the cluster Rydberg features at $<\mathrm{N}>=140$ (taken from the top spectrum in Figure 2, see text for details). 


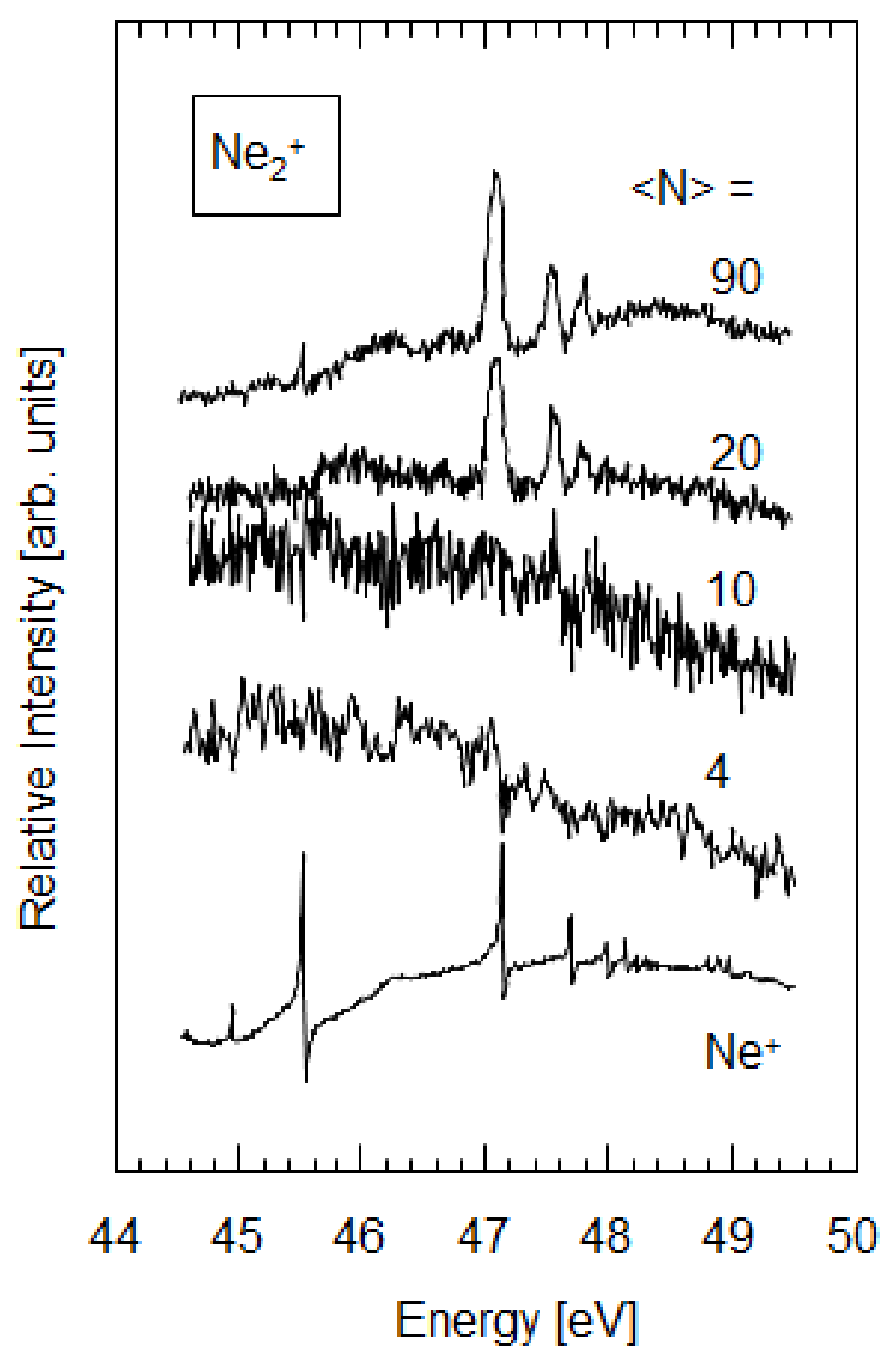

Figure 1 


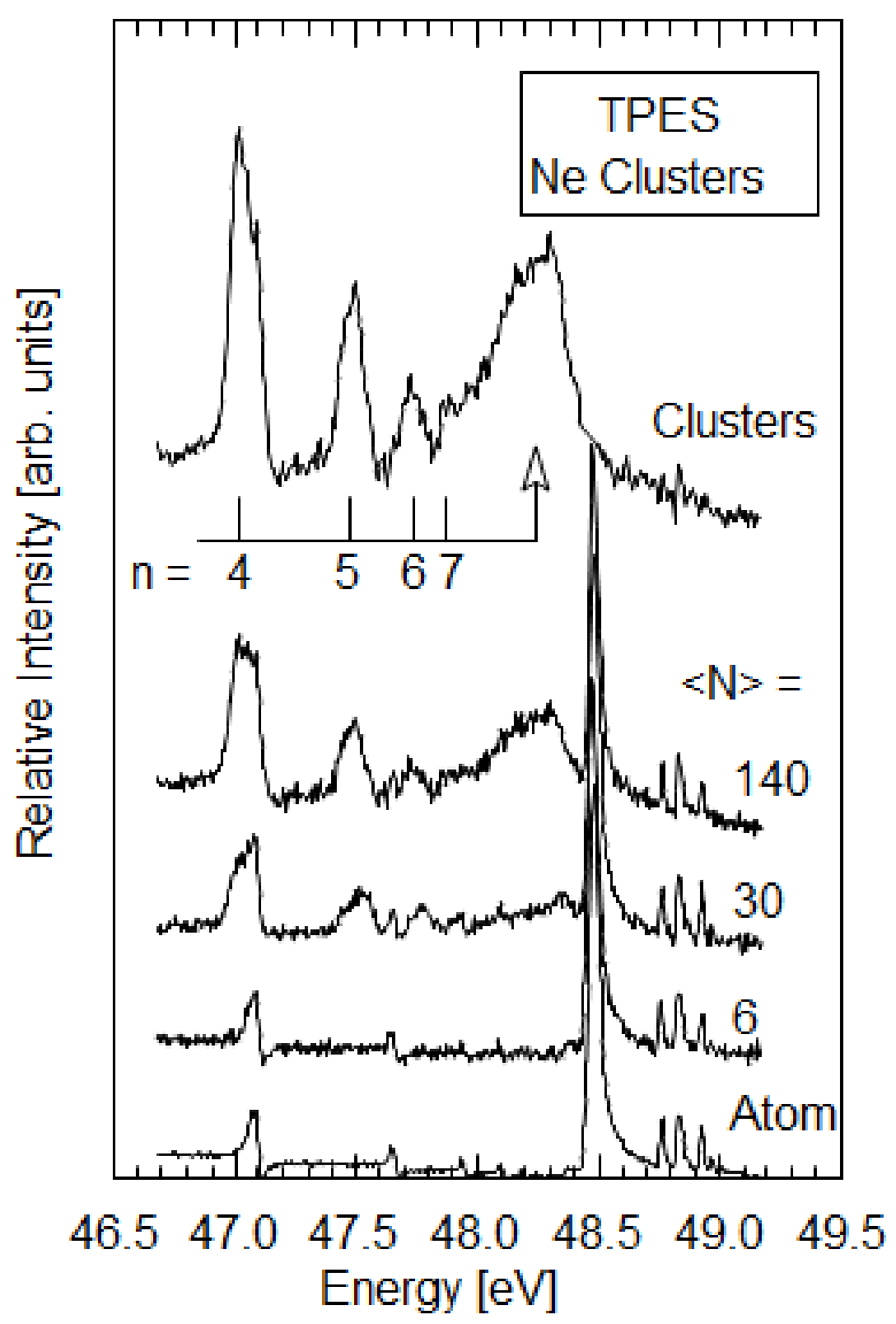

Figure 2 


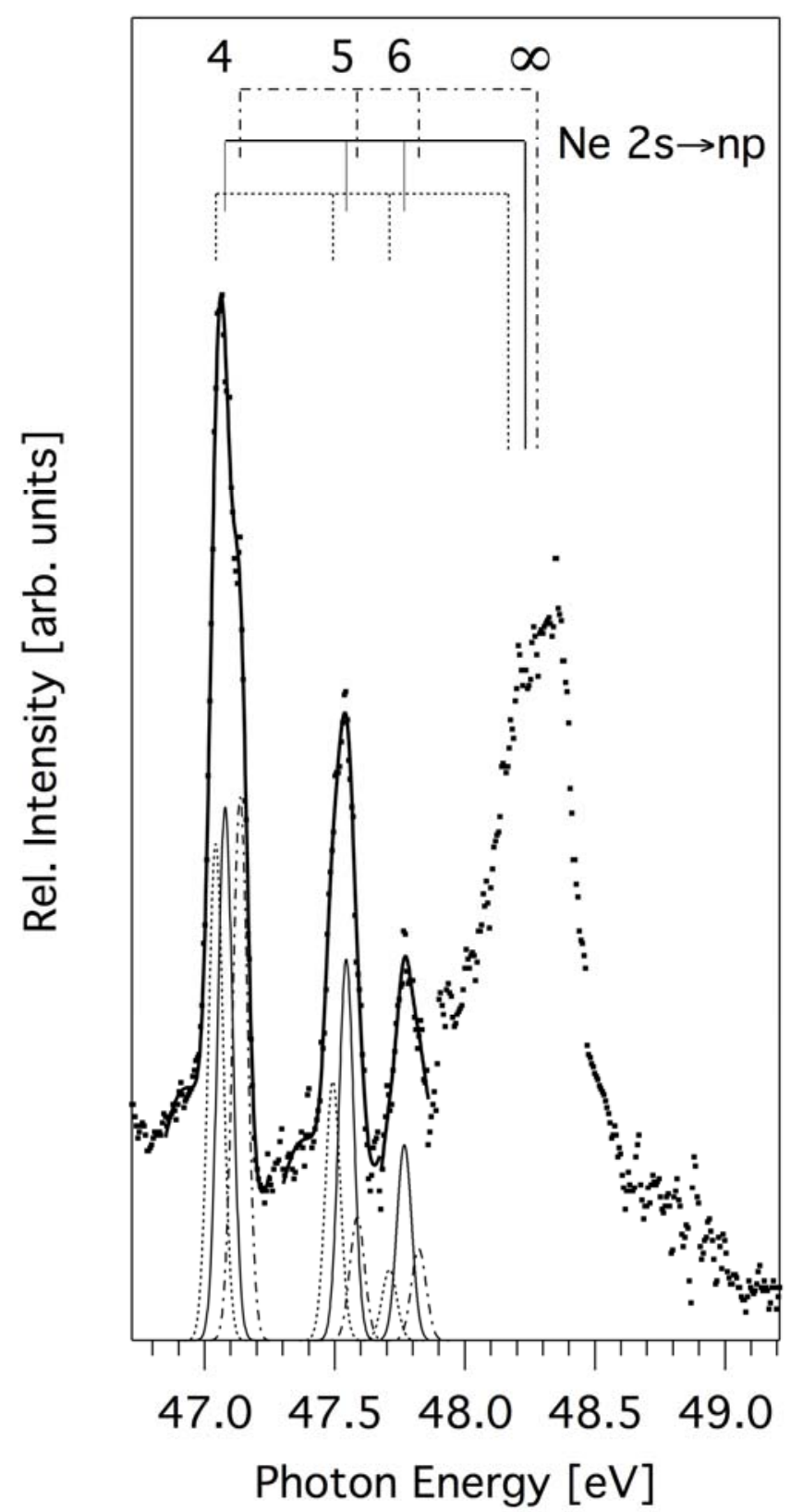

Figure 3 